\title{
Application of 3D-Arterial Spin Labeling Brain Perfusion Imaging in the Preoperative Diagnosis and Grading of Glioma
}

\author{
YU TING LV, XIAO XING MING ${ }^{1}$, SHAO XING LV, DAO HONG MING*AND FENG ZHU1 \\ Department of Radiology, ${ }^{1}$ Department of Neurology, People's Hospital of Yangxin County, Huangshi, Hubei, China
}

Lv et al.: 3D-ASL Brain Perfusion Imaging in the Preoperative Diagnosis and Grading of Glioma

\begin{abstract}
To explore the value of three-dimensional arterial spin labeling brain perfusion imaging in the preoperative diagnosis and grading of glioma. A total of 128 patients with preoperative and postoperative intracranial occupying lesions and glioma between January 2018 and December 2020, including 76 preoperative patients and 52 postoperative patients. Routine magnetic resonance imaging and three-dimensional arterial spin labeling perfusion examinations were performed in all patients, and the cerebral blood flow values and the area size of the lesions were measured preoperatively and postoperatively, and the ratio of the two was calculated to standardize the regional cerebral blood flow. The perfusion of three-dimensional arterial spin labeling in glioma was characterized by high perfusion in solid areas of high-grade glioma and low perfusion in solid areas of low-grade glioma. The correct diagnostic rate of perfusion grading in threedimensional arterial spin labeling was found to be $93.21 \%$, while the accuracy rate of magnetic resonance imaging was only $84.33 \%$, using pathological diagnostic results as the standard. The difference between the two was statistically significant $(\mathbf{p}<\mathbf{0 . 0 5})$. The results showed that the tumor cerebral blood flow was high in the high-grade glioma group compared with the low-grade glioma group $(p<0.05)$. The difference between the two was statistically significant $(p<0.05)$. Regional cerebral blood flow values were statistically significant $(p<0.05)$ between high-grade and low-grade gliomas. Receiver operating characteristic curve analysis showed that at preoperative diagnosis, regional cerebral blood flow $=2.157$. The sensitivity and specificity were $94.8 \%$ and $93.2 \%$, respectively; while in postoperative diagnosis, the optimal diagnostic threshold for regional cerebral blood flow value to predict postoperative glioma recurrence was 3.27, with a sensitivity and specificity of $65.78 \%$ and $90.31 \%$, respectively. The difference in lesion area between cerebral blood flow perfusion images of low-grade gliomas and those measured by conventional enhanced magnetic resonance imaging was statistically significant $(p<0.05)$. The difference between cerebral blood flow perfusion images of high-grade gliomas and the lesion area measured by conventional enhanced magnetic resonance imaging was not statistically significant $(p>0.05)$. In the diagnosis of postoperative recurrence, the cerebral blood flow values of lesions in the glioma recurrence group were significantly higher than those in the glioma non-recurrence group. The regional cerebral blood flow values of lesions in the glioma recurrence group were significantly higher than those in the glioma non-recurrence group, and the differences between the two groups were statistically different $(p<0.05)$. Three-dimensional arterial spin labeling can provide a more comprehensive and accurate assessment of glioma, which provides a new theoretical basis for preoperative grading of glioma, prediction of postoperative recurrence and assessment of surgical planning.
\end{abstract}

Key words: Glioma, magnetic resonance imaging, three-dimensional arterial spin labeling, diagnostic grading, cerebral blood flow

Gliomas, also known as brain stem tumors, account for $37 \%$ to $52 \%$ of the total intracranial tumors ${ }^{[1]}$, of which astrocytoma and polar glioblastoma are the most common $^{[2]}$. More than $75 \%$ of gliomas are malignant, also known as high-grade gliomas. High-grade gliomas are more common in adolescents and middle-aged, mainly in $\operatorname{men}^{[3,4]}$. At present, the resection range of glioma will be expanded, and accurate determination of the surgical range can better protect brain function and reduce the late complications ${ }^{[5]}$. Therefore, it is of great

*Address for correspondence

E-mail: ding202012@163.com 
clinical significance to find more rapid and accurate indicators for preoperative evaluation.

At present, imaging examination are the best clinical diagnostic methods for high-grade gliomas, including magnetic resonance imaging (MRI), magnetic resonance spectroscopy and computed tomography (CT). Among them, MRI has the characteristics of high resolution and multi axial imaging, but there are obvious deficiencies in the evaluation of preoperative pathological grading, recurrence after treatment and peritumoral brain tissue infiltration. Three-dimensional arterial spin labeling (3D-ASL) is a kind of cerebral perfusion imaging technology which uses the blood in patients as contrast agent ${ }^{[6]}$. It has been reported that $3 \mathrm{D}$-ASL has potential value in grading diagnosis and prognosis evaluation of thyroid cancer and breast cancer $^{[7,8]}$, and also has certain application value in glioma grading and residual lesion identification ${ }^{[9,10]}$. Therefore, in order to explore the application value of $3 \mathrm{D}-\mathrm{ASL}$ in the preoperative diagnosis and classification of glioma, the clinical and 3D-ASL examination data of 128 patients with glioma who underwent surgery and were confirmed by pathology in the past $2 \mathrm{y}$ were analyzed retrospectively.

\section{MATERIALS AND METHODS}

\section{General information:}

Patients with intracranial space occupying lesions and treated with glioma surgery, who were initially diagnosed or admitted to the ward from January 2018 to December 2020 were collected.

Inclusion criteria of preoperative group: The metastatic tumor was diagnosed by clinical follow-up for many times, and the imaging manifestations were multiple intracranial lesions, and there was postoperative pathology of primary lesions; except for brain metastases, other brain tumors were finally confirmed by pathology.

Inclusion criteria of postoperative group: Treated with complete or partial resection of gliomas (confirmed by pathology); during the follow-up, abnormal enhancement lesions or abnormal high perfusion areas appeared in the original lesion area and surrounding areas. According to World Health Organization (WHO) brain tumor classification standard, grade I and II were classified as low-grade glioma, and grade III and IV were classified as high-grade glioma. Finally, 128 patients met the diagnostic criteria. The preoperative group included 76 cases, 41 males and 35 females, aged $18-74$ y (mean $49.82 \pm 3.25$ y). The postoperative group included 52 cases, 31 males and 21 females, aged $19-72$ y (mean $47.96 \pm 2.40 \mathrm{y}$ ).

\section{Equipment:}

General Electric (GE) Discovery Magnetic resonance (MR) 750 3.0T MRI scanner, GE MR Advanced work station (ADW) 4.6 workstation and 8-channel phased array coil were used. The contrast medium was paramagnetic contrast agent ((gadoliniumdiethylenetriamine pentaacetic acid (Gd-DTPA)) and gadopentetate Meglumine injection. All patients underwent head MRI scan, followed by 3D-ASL sequence (including 3DT1BRAV0 positioning image) and enhanced scan. During the examination, both sides of the patient's head were filled and fixed with soft objects, and the patient was instructed to reduce head movement as much as possible.

\section{Scanning parameters:}

The first examination included MRI scan (T1-weighted fluid-attenuated inversion recovery (T1-FLAIR), T2FLAIR, T2-periodically rotated overlapping parallel lines with enhanced reconstruction (T2-PROPELLER), diffusion-weighted imaging (DWI) Asset) and enhanced scan and 3D-ASL examination. Gd-DTPA was used as contrast agent, the dose was $0.2 \mathrm{mmol} / \mathrm{kg}$, and the injection flow rate was $3 \mathrm{ml} / \mathrm{s}$. DWI scanning parameters: echo time (TE) $66 \mathrm{~ms}$, repetition time (TR) $5060 \mathrm{~ms}$, field-of-view (FOV) $30 \mathrm{~cm} \times 30 \mathrm{~cm}$, matrix size $200 \times 192$, layer thickness $5 \mathrm{~mm}$, layer spacing $1 \mathrm{~mm}, \mathrm{~b}$ value $0-1000 \mathrm{~s} / \mathrm{mm}^{2}$, total scanning time $225 \mathrm{~s}$, excitation times 8. 3 3D-ASL scanning parameters: TE $1.5 \mathrm{~ms}$, TR $4632 \mathrm{~ms}$, FOV $24 \mathrm{~cm} \times 24$ $\mathrm{cm}$, matrix size $96 \times 91$, layer thickness $4 \mathrm{~mm}$, delay $1535 \mathrm{~ms}$.

\section{Data processing:}

The original 3D-ASL image was imported into GE MR ADW 4.6 workstation, and the image was corrected, de noised and post processed by using fentool software package. By fusing the functional image and 3DT1BRAV0 anatomical image, the selected region of interest (ROI) and enhanced scan image were confirmed in the same position. Finally, the 3D-ASL cerebral blood flow diagram was obtained. The cerebral blood flow (CBF) values of the maximum perfusion area and the contralateral mirror area of the tumor as well 
as the corresponding $\mathrm{CBF}$ values of the contralateral brain tissue were measured, and calculated the ratio of the two, named standardized tumor blood flow (regional cerebral blood flow-rCBF). In order to correct the difference of mean cerebral blood flow perfusion caused by age dependence and patient dependence, the contralateral mirror region was used as the reference region. Quantitative analysis was performed by the following formula: the $\mathrm{CBF}$ value of the tumor divided by the $\mathrm{CBF}$ value of the contralateral mirror ROI to obtain the rCBF value. Conventional sequence and 3D-ASL perfusion images were analyzed by two experienced MRI physicians.

The area of the lesion was measured. Firstly, the boundary of the lesion was delineated on the perfusion functional map of the largest interface of glioma. Repeat the measurement for 3 times, and take the average value as the lesion area on the perfusion image. Secondly, the area of lesions on the corresponding layers of conventional enhanced scanning images was measured. Repeat the measurement for 3 times, and take the average value as the area of the lesion on the conventional enhanced scanning image.

\section{Follow up:}

6 mo after operation, the patients were hospitalized for MRI scan. According to the recurrence, the patients were divided into recurrence group and non-recurrence group, and the rCBF of the two groups were compared. Recurrence criteria: compared with MRI at the time of discharge, new space occupying lesions or density changes of brain parenchyma were found at the operation site, and confirmed by pathology again as glioma.

\section{Observed indexes:}

The signal characteristics and morphology of the lesions were observed by two radiologists independently. CBF value measurement: ROI was selected to be placed in the cross section of $20 \sim 40 \mathrm{~mm}^{2}$. In order to reduce the influence of human factors as much as possible, each lesion was measured three times and the average value was taken; $\mathrm{CBF}$ value of tumor entity and contralateral hemisphere was measured. The ROI of the tumor body was selected from three brain parenchyma regions with the most obvious enhancement in the lesion and the opposite side of the lesion was relatively normal. The $\mathrm{CBF}$ average value was calculated after measurement. Then divide it by the CBF of the contralateral hemisphere to obtain the $\mathrm{rCBF}$ of the perfusion image. The rCBF between 0.9 and 1.1 was defined as normal perfusion, and $\mathrm{rCBF}>1.1$ represented high perfusion, and $\mathrm{rCBF}<0.9$ represented low perfusion. Pay attention to avoid blood vessel, hemorrhage, necrosis and cystic change in the measurement.

\section{Statistical analysis:}

All data were processed on SPSS 20.0 statistical software, and the results were expressed as (mean \pm standard deviation (SD)). The normality of rCBF data was tested. If the distribution was normal, independent sample $t$ test was performed. Chi square test was used to compare the diagnostic accuracy of conventional MRI sequences and 3D-ASL perfusion imaging in the qualitative and grading diagnosis of gliomas. $\mathrm{p}<0.05$ meant the difference was statistically significant.

\section{Comparison of accuracy rate of 3D-ASL and MRI in grading glioma:}

The perfusion characteristics of 3D-ASL in gliomas were as follows: high blood flow perfusion was found in the solid areas of high-grade gliomas while low blood flow perfusion in the solid areas of low-grade gliomas. According to WHO grading principle of glioma, based on the perfusion characteristics of 3D-ASL, there were 18 cases of low-grade gliomas, including 6 cases of grade I and 12 cases of grade II, and there were 58 cases of high-grade gliomas, including 37 cases of grade III and 21 cases of grade IV. Conventional MRI identified 9 cases of grade I, 14 cases of grade II, 36 cases of grade III and 19 cases of grade IV. Take the pathological diagnosis as the standard, the accuracy rate of 3D-ASL perfusion classification was $93.21 \%$, while that of MRI was only $84.33 \%$. The difference was statistically significant $(\mathrm{p}<0.05)$, as shown in Table 1 .

TABLE 1: COMPARISON OF ACCURACY RATE OF 3D-ASL AND MRI IN GRADING GLIOMA

\begin{tabular}{lccccc}
\hline Examination methods & Grade I & Grade II & Grade III & Grade IV & Accuracy rate \\
\hline MRI & 9 & 14 & 36 & 19 & 84.33 \\
3D-ASL & 6 & 12 & 37 & 21 & 93.21 \\
Pathological diagnosis & 6 & 11 & 36 & 23 & - \\
$\mathrm{t}$ & & & & 8.321 \\
$\mathrm{p}$ & & & & $<0.001$ \\
\hline
\end{tabular}


CBF and $\mathrm{rCBF}$ values of 3D-ASL in patients with gliomas:

The results showed that $\mathrm{CBF}$ value of high-grade gliomas group was higher than that of low-grade gliomas group, and the difference was statistically significant $(\mathrm{p}<0.05)$. There were significant differences in rCBF value between high-grade gliomas and lowgrade gliomas $(\mathrm{p}<0.05)$, as shown in Table 2 .

\section{Comparison of ROC curve analysis of $\mathrm{rCBF}$ values before and after operation:}

ROC curve was used to obtain the best threshold value of $\mathrm{rCBF}$ for distinguishing high-grade and low-grade gliomas. ROC curve analysis showed that at preoperative diagnosis, when $\mathrm{rCBF}=2.157$, the sensitivity and specificity were $94.8 \%$ and $93.2 \%$, respectively; while at postoperative diagnosis, the optimal diagnostic threshold for rCBF value to predict postoperative glioma recurrence was 3.27 , with a sensitivity and specificity of $65.78 \%$ and $90.31 \%$, respectively, as shown in Table 3.

\section{Comparison of tumor area measured by MRI and 3D-ASL perfusion:}

The results showed that there was significant difference in the area of high-grade gliomas between 3D-ASL perfusion image and conventional enhanced MRI $(p<0.05)$. There was no difference in the area of lowgrade gliomas between 3D-ASL perfusion image and conventional enhanced MRI ( $p>0.05)$, as shown in Table 4.

TABLE 2: CBF AND RCBF VALUES OF 3D-ASL IN PATIENTS WITH GLIOMAS

\begin{tabular}{lccc}
\hline Group & $\mathrm{N}$ & $\mathrm{CBF}$ value & rCBF value \\
\hline High-grade gliomas & 59 & $117.004 \pm 56.330$ & $3.774 \pm 1.569$ \\
Low-grade gliomas & 17 & $36.452 \pm 11.237$ & $0.731 \pm 0.254$ \\
$\mathrm{t}$ & & 13.765 & 14.005 \\
$\mathrm{p}$ & & $<0.001$ & $<0.001$ \\
\hline
\end{tabular}

TABLE 3: ROC CURVE ANALYSIS OF rCBF VALUES

\begin{tabular}{lccc}
\hline Group & $\begin{array}{c}\text { The optimal } \\
\text { diagnostic } \\
\text { threshold for } \\
\text { rCBF value }\end{array}$ & Sensitivity & Specificity \\
\hline Before operation & 2.157 & $94.8 \%$ & $93.2 \%$ \\
After operation & 3.27 & $65.78 \%$ & $90.31 \%$ \\
\hline
\end{tabular}

CBF and $\mathrm{rCBF}$ values of 3D-ASL in patients with recurrent gliomas after operation:

The CBF values of lesions in the glioma recurrence group were significantly higher than those in the glioma non-recurrence group. The rCBF values of lesions in the glioma recurrence group were significantly higher than those in the glioma non-recurrence group, and the differences were statistically different $(p<0.05$, respectively), as shown in Table 5.

\section{RESULTS AND DISCUSSION}

Gliomas are mostly chronic onset and gradually aggravating. The clinical manifestations are diverse and complex, and the severity varies greatly. Some patients only showed dizziness and headache, while in severe cases, red nucleus tremor and paroxysmal coma may occur. High-grade gliomas (WHO grade 3-4) are poorly differentiated gliomas, which are malignant with poor survival prognosis ${ }^{[9]}$. Radiotherapy and drug therapy can prolong the life of patients ${ }^{[10-12]}$. The accurate diagnosis of glioma and its stage has a good guiding role in the later treatment.

3D-ASL is a completely noninvasive, quantitative measurement of $\mathrm{CBF}$ of magnetic resonance perfusion examination. As 3D-ASL has the advantages of no ionizing radiation, good repeatability, and is not affected by the damage of blood-brain barrier, calcification, bleeding and skull base structure, and has no restriction on the application of exogenous contrast agents (patients with renal failure, etc.), so it can obtain the most accurate hemodynamic parameters of human body in its original physiological state, and can monitor the perfusion changes in a long-term follow-up. In 3D-ASL, water proton in arterial blood was used as endogenous contrast agent, and there was no exogenous contrast agent involved. There was no possibility that contrast agent penetrated into the lesion and surrounding edema area due to the damage of blood-brain barrier, which led to underestimate of the perfusion information of the lesion itself. Besides, the patient's renal function does not need to be considered in 3D-ASL. Previous studies have confirmed the feasibility of 3D-ASL in the evaluation of brain tumors. Tourdia et al. found that 3D-ASL perfusion imaging can be used to diagnose and grade glioma ${ }^{[13]}$. Kim et al. found that 3D-ASL

TABLE 4: COMPARISON OF TUMOR AREA MEASURED BY MRI AND 3D-ASL PERFUSION

\begin{tabular}{lcccccc}
\hline Group & N & 3D-ASL & MRI & difference & t & P \\
\hline High-grade gliomas & 39 & $1324.73 \pm 115.39$ & $1543.68 \pm 111.29$ & $-219.05 \pm 98.47$ & 3.767 & 0.013 \\
Low-grade gliomas & 13 & $1652.45 \pm 107.47$ & $1687.27 \pm 118.54$ & $-34.82 \pm 73.44$ & 1.431 & 0.073 \\
\hline
\end{tabular}


TABLE 5: CBF AND RCBF VALUES OF 3D-ASL IN PATIENTS WITH RECURRENT GLIOMAS AFTER OPERATION

\begin{tabular}{lccc}
\hline Group & $\mathrm{N}$ & $\mathrm{CBF}$ & $\mathrm{rCBF}$ \\
\hline Glioma recurrence group & 39 & $99.78 \pm 37.29$ & $2.971 \pm 0.890$ \\
Glioma non-recurrence & 13 & $31.66 \pm 17.33$ & $0.625 \pm 0.343$ \\
group & & 24.372 & 18.809 \\
$\mathrm{t}$ & & $<0.001$ & $<0.001$ \\
$\mathrm{p}$ & & & \\
\hline
\end{tabular}

can improve the accuracy of preoperative grading of glioma $^{[14]}$.

The perfusion images and perfusion parameters of 76 cases of brain tumors were analyzed by 3D-ASL and conventional enhancement techniques. The results showed that $\mathrm{CBF}$ and $\mathrm{rCBF}$ values were significantly different between high-grade astrocytoma and lowgrade glioma $(\mathrm{p}<0.05)$. ROC curve analysis showed that at preoperative diagnosis, when $\mathrm{rCBF}=2.157$, the sensitivity and specificity were $94.8 \%$ and $93.2 \%$, respectively. These results indicate that $3 \mathrm{D}-\mathrm{ASL}$ can display the changes of blood perfusion of tumor, and it is helpful for preoperative evaluation and grading diagnosis of brain tumors.

In this study, we compared the tumor cross-sectional area measured by conventional enhanced images and ASL perfusion images of each patient. The results showed that there was significant difference in the area of high-grade gliomas between 3D-ASL perfusion image and conventional enhanced MRI $(p<0.05)$. There was no difference in the area of low-grade gliomas between 3D-ASL perfusion image and conventional enhanced MRI $(p>0.05)$. The above results are considered to be due to the invasive growth of glioma. Tumor cells usually infiltrate along the vascular channels of the white matter tracts and easily invade the associated fibers. This kind of infiltration and growth mode shows that the tumor does not damage the bloodbrain barrier while infiltrating into the distance, and the corresponding nerve cell structure remains intact ${ }^{[15]}$. 3D-ASL perfusion image can more accurately evaluate the blood supply of the tumor, which has important guiding significance for the prevention of surgical risk. It has been reported that the $\mathrm{rCBF}$ of peritumoral edema in some gliomas is higher than that in normal brain tissues, indicating the presence of tumor infiltration. Law et $a l .^{[16]}$ showed that the rCBF of high-grade peritumoral edema was significantly higher than that of the contralateral white matter, and the rCBF of the peritumoral edema area was higher than that of the peritumoral edema area away from the tumor, which may be due to the less tumor infiltration in the area far away from the tumor edema. Therefore, compared with conventional MRI images, 3D-ASL can display the tumor boundary more accurately, and has great value in making operation plan and selecting radiotherapy target area.

Clinically, the treatment plan for glioma recurrence and gliosis is different, so it is very important to accurately identify the two for the evaluation of curative effect and prognosis after glioma surgery. However, the traditional imaging examination is limited to observe the changes of operation area and brain tissue edema, and cannot effectively distinguish the residual or recurrent tumor ${ }^{[17,18]}$. The recurrence of gliomas after surgery usually originates from the tumor cells in situ and visible under microscope outside the surgical margin. Usually, the imaging features of recurrent tumors are similar to those before operation. When MRI showed no abnormal signal in T2-weighted imaging (T2WI) of brain parenchyma around the operating table, while T1-weighted imaging (T1W1) did not enhance on conventional enhanced scan, it was considered that there was no tumor recurrence in situ. However, when the range of postoperative recurrence of unreinforced tumors (low-grade gliomas) was small, it was difficult to distinguish them from postoperative and radiotherapy reactions and it was difficult to be detected. Some unreinforced gliomas may show enhancement when they recurred in situ. When the enhanced tumor recurred, MRI showed nodular, patchy, banded, annular or lumpy enhancement, which was difficult to distinguish from radiation-induced brain necrosis. Therefore, other medical imaging methods with high specificity and sensitivity were needed to identify. In present study, the rCBF of patients with in situ recurrence of glioma was significantly higher than that of patients without recurrence, suggesting that the increase of vascular density and blood perfusion in the local area of recurrent glioma may be related to the increased expression of angiogenic factor and tumor growth factor and the increase of capillary angiogenesis ${ }^{[19,20]}$. The early invasion and metastasis of tumor cells depend on blood vessels, so the brain tissue near the tumor parenchyma is more prone to tumor cell infiltration. In our study, the optimal diagnostic threshold for $\mathrm{rCBF}$ value to predict postoperative glioma recurrence was 3.27 , with a sensitivity and specificity of $65.78 \%$ and $90.31 \%$, respectively, which indicated that 3D-ASL has a good application value in the diagnosis of recurrent glioma. 
In conclusion, 3D-ASL can provide a more comprehensive and accurate evaluation of glioma, and provide a new theoretical basis for the evaluation of preoperative grading, prediction of postoperative recurrence and formulation of surgical plan.

\section{Acknowledgements:}

This work was supported by the People's Hospital of Yangxin County, Yu Ting LV and Xiao Xing Ming are contributed equally to this work and considered co-first authors. D. H. Ming and F. Zhu are considered co-corresponding authors.

\section{Conflict of Interests:}

The authors declared no conflict of interest.

\section{REFERENCES}

1. Eichler AF, Batchelor TT. Primary central nervous system lymphoma: presentation, diagnosis, and staging. Neurosurg Focus 2006;21(5):1-9.

2. Yamasaki F, Kurisu K, Satoh K, Arita K, Sugiyama K, Ohtaki $\mathrm{M}$, et al. Apparent diffusion coefficient of human brain tumors at MR imaging. Radiology 2005;235(3):985-91.

3. Chiang IC, Hsieh TJ, Chiu ML, Liu GC, Kuo YT, Lin WC. Distinction between pyogenic brain abscess and necrotic brain tumour using 3-tesla MR spectroscopy, diffusion and perfusion imaging. Br J Radiol 2009;82(982):813-20.

4. Ohba S, Ushioda T, Nakagawa T, Shimizu K, Murakami H. Diffusion magnetic resonance imaging for enhanced visualization of malignant cerebral tumors and abscesses. Neurol India 2011;59(5):674-8.

5. Hrabe J, Lewis DP. Two analytical solutions for a model of pulsed arterial spin labeling with randomized blood arrival times. J Magn Reson 2004;167(1):49-55.

6. Zaharchuk G. Theoretical basis of hemodynamic MR imaging techniques to measure cerebral blood volume, cerebral blood flow, and permeability. AJNR Am J Neuroradiol 2007;28(10):1850-8.

7. Wolf RL, Detre JA. Clinical neuroimaging using arterial spin-labeled perfusion magnetic resonance imaging. Neurotherapeutics 2007;4(3):346-59.

8. Warmuth C, Gunther M, Zimmer C. Quantification of blood flow in brain tumors: comparison of arterial spin labeling and dynamic susceptibility-weighted contrast-enhanced MR imaging. Radiology 2003;228(2):523-32.

9. Knutsson L, van Westen D, Petersen ET, Bloch KM, Holtas $\mathrm{S}$, Stahlberg F, et al. Absolute quantification of cerebral blood flow: correlation between dynamic susceptibility contrast MRI and model-free arterial spin labeling. Magn Reson Imaging 2010;28(1):1-7.

10. Detre JA, Alsop DC. Perfusion magnetic resonance imaging with continuous arterial spin labeling: methods and clinical applications in the central nervous system. Eur $\mathrm{J}$ Radiol 1999;30(2):115-24.

11. Golay X, Hendrikse J, Lim TC. Perfusion imaging using arterial spin labeling. Top Magn Reson Imaging 2004;15(1):10-27.

12. Martinez-Martinez A, Martinez-Bosch J. Perfusion magnetic resonance imaging for high grade astrocytomas: can cerebral blood volume, peak height, and percentage of signal intensity recovery distinguish between progression and pseudo progression. Radiologia 2014;56(1):35-43.

13. Bisdas S, Naegele T, Ritz R, Dimostheni A, Pfannenberg C, Reimold $\mathrm{M}$, et al. Distinguishing recurrent high-grade gliomas from radiation injury: a pilot study using dynamic contrastenhanced MR imaging. Acad Radiol 2011;18(5):575-83.

14. Wen PY, Macdonald DR, Reardon DA, Cloughesy TF, Sorensen AG, Galanis E, et al. Updated response assessment criteria for high-grade gliomas: response assessment in neurooncology working group. J Clin Oncol 2010;28(11):1963-72.

15. Matsusue E, Fink JR, Rockhill JK, Ogawa T, Maravilla KR. Distinction between glioma progression and post-radiation change by combined physiologic MR imaging. Neuroradiology 2010;52(4):297-306.

16. Robson PM, Dai W, Shankaranarayanan A, Rofsky NM, Alsop DC. Time-resolved vessel-selective digital subtraction MR angiography of the cerebral vasculature with arterial spin labeling. Radiology 2010;257(2):507-15.

17. Wu WC, Fernandez-Seara M, Detre JA, Wehrli FW, Wang J. A theoretical and experimental investigation of the tagging efficiency of pseudocontinuous arterial spin labeling. Magn Reson Med 2007;58(5):1020-7.

18. White CM, Pope WB, Zaw T, Qiao J, Naeini KM, Lai A, et al. Regional and voxel-wise comparisons of blood flow measurements between dynamic susceptibility contrast magnetic resonance imaging (DSC-MRI) and arterial spin labeling (ASL) in brain tumors. J Neuroimaging 2014;24(1):2330.

19. $\mathrm{Hu}$ LS, Baxter LC, Smith KA, Feuerstein BG, Karis JP, Eschbacher JM, et al. Relative cerebral blood volume values to differentiate high-grade glioma recurrence from posttreatment radiation effect: direct correlation between image-guided tissue histopathology and localized dynamic susceptibility-weighted contrast-enhanced perfusion MR imaging measurements. AJNR Am J Neuroradiol 2009;30(3):552-8.

20. Ellika SK, Jain R, Patel SC, Scarpace L, Schultz LR, Rock JP, et al. Role of perfusion CT in glioma grading and comparison with conventional MR imaging features. AJNR Am J Neuroradiol 2007;28(10):1981-7.

This is an open access article distributed under the terms of the Creative Commons Attribution-NonCommercial-ShareAlike 3.0 License, which allows others to remix, tweak, and build upon the work non-commercially, as long as the author is credited and the new creations are licensed under the identical terms

This article was originally published in a special issue, "Clinical Research in Pharmaceutical and Biomedical Sciences" Indian J Pharm Sci 2021:83(1)Spl issue1;83-88 